\title{
A New Vibration Absorber Design for Under-Chassis Device of a High-Speed Train
}

\author{
Yu Sun, Jinsong Zhou, Dao Gong, Wenjing Sun, and Zhanghui Xia
}

Institute of Rail Transit, Tongji University, Shanghai, China

Correspondence should be addressed to Jinsong Zhou; jinsong.zhou@tongji.edu.cn

Received 1 June 2017; Revised 27 August 2017; Accepted 12 September 2017; Published 17 October 2017

Academic Editor: Francesco Ripamonti

Copyright ( 2017 Yu Sun et al. This is an open access article distributed under the Creative Commons Attribution License, which permits unrestricted use, distribution, and reproduction in any medium, provided the original work is properly cited.

\begin{abstract}
To realize the separation of vertical and lateral stiffness of the under-chassis device, a new type of vibration absorber is designed by using the negative stiffness of the disc spring in parallel with the rubber component. To solve its transmission characteristics, harmonic transfer method was used. A rigid-flexible coupling multibody dynamic model of a high-speed train with an elastic car body is established, and the vertical and lateral optimal stiffness of the under-chassis device are calculated. The Sperling index and acceleration PSD of the vehicle with the new vibration absorber and the vehicle with traditional rubber absorber are compared and analyzed. The results show that, with the new vibration absorber, vehicle's running stability and vibration of the car body are more effective than the vehicle with the traditional rubber absorber.
\end{abstract}

\section{Introduction}

The elastic vibration of car body is a serious problem as the increasingly speed and lightweight design of car body. For high-speed electric multiple units (EMUs), traction transformers, traction converters, and other devices are used to suspend under chassis of the car body. Some of them weigh more than 3 tons and include vibration sources. If they are hanging inappropriately, the vibration of the car body and the ride quality may deteriorate. Many researchers are focusing on how to suspend the under-chassis devices reasonably.

Carlbom [1] researched the structural flexibility of the car body by simulations and measurements and pointed out that the structural flexibility of the car body must be taken into account when predicting vertical vibration comfort. Foo and Goodall [2] studied the vibration reduction results of "skyhook damping" based on the active control theory. Diana et al. [3] presented a mathematical model which takes the deformability of car body and wheelset into account for the simulation of dynamic behaviour of a train running on a track, and the model allows reproducing the traintrack dynamic interaction in the whole frequency range from $0 \mathrm{~Hz}$ to $200 \mathrm{~Hz}$. Young and $\mathrm{Li}$ [4] modeled the car body and sleepers as finite length Timoshenko beams and rail as an infinite Timoshenko beam with discrete supports to investigate the effect of the vehicle speed on the response of the vehicle and track systems by Newmark method. Sun et al. [5] concluded that using vibration isolation design of vibration isolation equipment under the original parameters, the proposed high-quality equipment near the middle of the body suspension is more conducive to reducing the body vibration. Gong et al. [6] consider the body as support in the second suspension on the homogeneous Euler beam and the vehicle under the device design for dynamic vibration absorber. To suspend the device under the vehicle, using the dynamic vibration absorber principle, which can greatly reduce the body vibration, is proposed.

However, most of the studies rarely consider the vertical and lateral stiffness of the under-chassis devices but instead just consider the vertical stiffness. The main reason for this is that there is a fixed vertical-lateral stiffness ratio of the rubber elements used for the suspension of the under-chassis devices. The lateral stiffness can be determined once the vertical stiffness is determined. Aiming to fix this problem, this paper designs a new vibration absorber, which by using the negative stiffness of the disc spring in parallel with the rubber component can realize the separation of the vertical and lateral stiffness of the vehicle. This paper first analyzes 


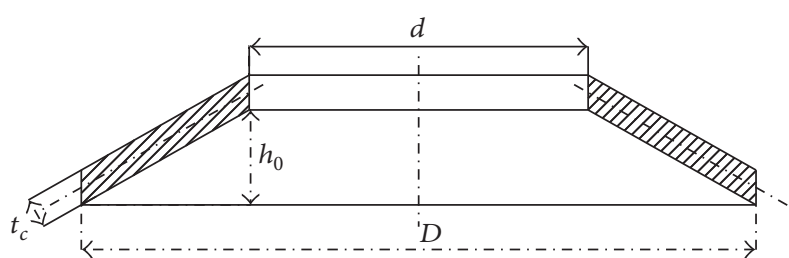

FIGURE 1: Cross-section of disc spring.

the force characteristics of the disc spring, introduces the design principle of the new vibration absorber, applies the harmonic excitation to the new vibration absorber, and uses the harmonic balance method to solve its transmission characteristics. Then based on the rigid-flexible coupling model of multibody dynamics, a new vibration absorber is designed for high-speed EMU and the optimum hanging frequency is obtained. Finally, in terms of acceleration power spectral density and running stability, the new vibration absorber is analyzed and compared with traditional rubber elements.

\section{Analysis on the Negative Stiffness of Disc Spring}

The cross-section of the disc spring is shown in Figure 1. In the figure, $t_{c}$ is the thickness, $h_{0}$ is the initial height, $D$ is the outer diameter, and $d$ is the inner diameter. Typically, the force of the disc spring without supporting surface (outsideinside diameter ratio $C=D / d=1 \sim 4$ ) can be expressed as [7]

$$
\begin{aligned}
F_{d}= & \frac{4 E}{\left(1-\mu^{2}\right)} \cdot \frac{t_{c}^{4}}{K_{1} D^{2}} \cdot \frac{x}{t_{c}} \\
& \times\left[\left(\frac{h_{0}}{t_{c}}-\frac{x}{t_{c}}\right)\left(\frac{h_{0}}{t_{c}}-\frac{x}{2 t_{c}}\right)+1\right],
\end{aligned}
$$

where $E$ is elastic modulus and $\mu$ is Poisson ratio. $x$ is the deformation of the disc spring from the initial position in vertical, and $K_{1}$ is related to $C$ :

$$
K_{1}=\frac{1}{\pi} \cdot \frac{((C-1) / C)^{2}}{((C+1) /(C-1))-2 / \ln C} .
$$

In order to study the disc spring's negative stiffness, let $\lambda=4 E /\left(1-\mu^{2}\right) \cdot t_{c}{ }^{4} / K_{1} D^{2}, x^{*}=x / t_{c}, \alpha=h_{0} / t_{c}$, and then $F_{d}$ is

$$
F_{d}=\lambda\left[\frac{1}{2} x^{* 3}-\frac{3}{2} \alpha x^{* 2}+\left(\alpha^{2}+1\right) x^{*}\right]
$$

derivative in terms of $x$; we can obtain

$$
k_{d}=\frac{\mathrm{d} F}{\mathrm{~d} x}=\frac{\lambda}{t_{c}}\left[\frac{3}{2} x^{* 2}-3 \alpha x^{*}+\alpha^{2}+1\right] .
$$

It can be seen that the stiffness $k_{d}$ of the disc spring is a quadratic function of the deformation $x$, the symmetry axis is $x=\alpha t_{c}$, and $k_{d}(0)=\lambda\left(\alpha^{2}+1\right) / t_{c}>0$, and to make the disc

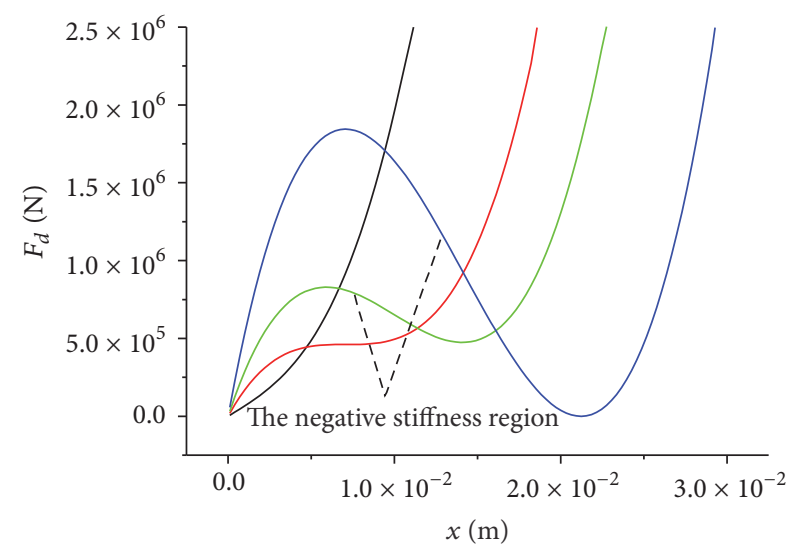

FIgURE 2: Disc spring characteristic curve.

spring have a negative stiffness region, $\Delta=(-3 \alpha)^{2}-6\left(\alpha^{2}+\right.$ 1) $>0$ must be satisfied, namely, $\alpha>\sqrt{2}$, so the negative stiffness region is

$$
\left(\frac{3 \alpha-\sqrt{3 \alpha^{2}-6}}{3}, \frac{3 \alpha+\sqrt{3 \alpha^{2}-6}}{3}\right) .
$$

According to (3), the force curve of the disc spring can be drawn, as shown in Figure 2. From the figure, disc spring will show different force characteristics according to its highthickness ratio $\alpha$. A negative stiffness region (region where the slope of the characteristic curve is negative) appears in the force characteristic when the high-thickness ration exceeds $\sqrt{2}$.

\section{Design Principle of New Vibration Absorber Based on Negative Stiffness}

Rubber spring is commonly used as elastic components in the suspension of under-chassis devices in high-speed EMUs. It can withstand multidirectional load and has a good absorption of high-frequency vibration. Rubber spring can be divided into compression type, shearing type, and compound type. Compressed rubber is usually used as the elastic component of under-chassis device, whose verticallateral stiffness ratio is usually above 4.5 [8]. This paper adopts the cylindrical rubber spring with the vertical-lateral stiffness ratio of 4.5 as the elastic component of the under-chassis device; the three-direction stiffness relation is $k_{z}=4.5 k_{y}$, $k_{x}=k_{y}$, where $k_{z}$ is vertical stiffness, $k_{y}$ is lateral stiffness, and $k_{x}$ is longitudinal stiffness.

In the past, only the vertical stiffness was considered in the elastic suspension design for the under-chassis devices of high-speed EMUs. The lateral and longitudinal stiffness are determined when the rubber spring is designed with the vertical stiffness as the goal. In fact, when only the vertical stiffness was taken into consideration, the lateral vibration cannot achieve the optimal situation. Therefore, it is necessary to consider the vertical and lateral hanging stiffness of the under-chassis device simultaneously. In order to solve this problem, a new vibration absorber is designed 


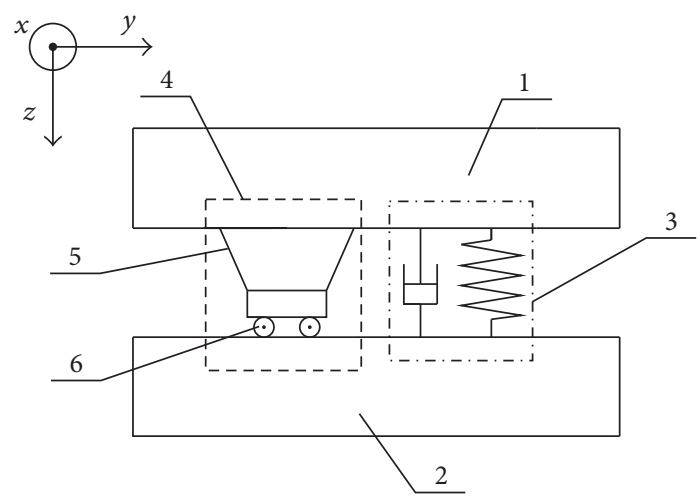

FIGURE 3: Principle diagram of vertical stiffness separation design (1: body connection end, 2: device connection end, 3: rubber spring, 4: negative stiffness mechanism, 5: disc spring, and 6: rolling mechanism).

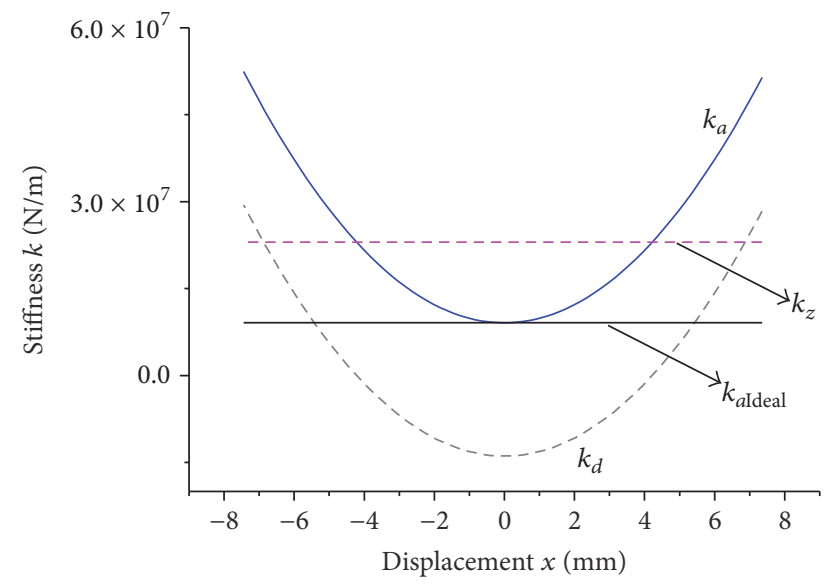

Figure 4: Vertical stiffness, displacement curve.

by connecting in parallel elements with positive and negative stiffness, where the positive stiffness is given by rubber spring and the negative stiffness is given by disc spring. The design steps are as follows: Firstly, the rubber spring is designed, so that the lateral stiffness is equal to the target value. Then, the disc spring is designed so that the value of its negative stiffness at the equilibrium position and the positive stiffness of the rubber spring which are added together is equal to the vertical stiffness target value. Finally, a rolling mechanism was designed to release the horizontal and lateral degrees of freedom of the disc spring, so that the horizontal and lateral stiffness of the disc spring are zero. Figure 3 shows the design principle diagram.

Figure 4 shows the relative vertical stiffness-displacement curve of the new vibration absorber stiffness, where $k_{a \text { Ideal }}$ is the target value of vertical stiffness, $k_{d}$ is the vertical stiffness of the disc spring which is calculated by (4), and $k_{a}=k_{z}+$ $k_{d}$ is the stiffness of the new vibration absorber. As can be seen from the figure, the vertical stiffness of the new vibration absorber $k_{a}$ can reach the stiffness design target value $k_{a \text { Ideal }}$ when the system vibration is in vicinity of the equilibrium position.

\section{Vertical Force Transmissibility Analysis of a New Vibration Absorber}

The restoring force $F$ of the new vibration absorber in vertical can be expressed as

$$
\begin{aligned}
F= & \frac{4 E}{\left(1-\mu^{2}\right)} \cdot \frac{t_{c}^{4}}{K_{1} D^{2}} \cdot \frac{x}{t_{c}} \\
& \times\left[\left(\frac{h_{0}}{t_{c}}-\frac{x}{t_{c}}\right)\left(\frac{h_{0}}{t_{c}}-\frac{x}{2 t_{c}}\right)+1\right]+k_{z} x .
\end{aligned}
$$

It includes the restoring force of the positive stiffness rubber spring and the restoring force of the negative stiffness disc spring. In order to solve the solution, coordinate transformation is needed; let

$$
\begin{aligned}
& u=x-h_{0}, \\
& \widehat{F}=\frac{F}{\left(k_{z} t_{c}\right)}, \\
& \beta=\frac{4 E t_{c}{ }^{3}}{\left(1-\mu^{2}\right) D^{2} K_{1} k_{z}}, \\
& \widehat{u}=\frac{u}{t_{c}} .
\end{aligned}
$$

The nondimensional is obtained as

$$
\widehat{F}=\widehat{u}+\alpha+\beta\left[\frac{1}{2} \widehat{u}^{3}+\left(1-\frac{1}{2} \alpha^{2}\right) \widehat{u}+\alpha\right] .
$$

The system is balanced when $\widehat{u}=0$, namely, $x=h_{0}$. At this time, the output force of the new vibration absorber is equal to the gravity of the under-chassis device, namely, $F\left(x=h_{0}\right)=m g$; substituting to the above equations, one can get

$$
(1+\beta) \alpha \cdot k_{z} t_{c}=m g \text {. }
$$

The equation of motion for harmonic forced excitation is given by

$$
m \ddot{u}+c \dot{u}+F-m g=F_{e} \cos (\omega t+\theta) .
$$

Nondimensionalising equation (10) gives

$$
\begin{aligned}
\widehat{u}^{\prime \prime} & +2 \varsigma u^{\prime}+\left(1+\beta-\frac{\beta}{2} \alpha^{2}\right) \widehat{u}+\frac{\beta}{2} \widehat{u}^{3} \\
& =\widehat{F}_{e} \cos (\Omega \tau+\theta),
\end{aligned}
$$

where $\omega_{n}=\sqrt{k_{z} / m}, \tau=\omega_{n} t_{c}, \Omega=\omega / \omega_{n}, \varsigma=c /\left(2 m \omega_{n}\right)$, $\widehat{F}_{e}=F_{e} /\left(k_{z} t_{c}\right)$, and $(\cdot)^{\prime}=\mathrm{d}(\cdot) / \mathrm{d} \tau$. Let $n_{1}=\left(1+\beta-(\beta / 2) \alpha^{2}\right)$, $n_{3}=\beta / 2$; it becomes

$$
\widehat{u}^{\prime \prime}+2 \varsigma \widehat{u}^{\prime}+n_{1} \widehat{u}+n_{3} \widehat{u}^{3}=\widehat{F}_{e} \cos (\Omega \tau+\theta) .
$$

This is a Duffing equation with force excitation. Apply the Harmonic Balance (HB) method and assume its solution as [9]

$$
\begin{aligned}
\widehat{u} & =A \cos (\Omega \tau), \\
\widehat{u}^{\prime} & =-A \Omega \sin (\Omega \tau), \\
\widehat{u}^{\prime \prime} & =-A \Omega^{2} \cos (\Omega \tau) .
\end{aligned}
$$


The frequency-amplitude relationship can be obtained

$$
\begin{aligned}
\left(-\Omega^{2} A\right. & \left.+\frac{3}{4} n_{1} A^{3}+n_{3} A\right) \cos (\Omega \tau)-2 \varsigma \Omega A \sin (\Omega \tau) \\
& +\frac{1}{4} n_{1} A^{3} \cos (3 \Omega \tau) \\
= & \widehat{F}_{e} \cos (\Omega \tau) \cos \theta+\widehat{F}_{e} \sin (\Omega \tau) \sin \theta
\end{aligned}
$$

With the term containing $\cos (3 \Omega \tau)$ neglected and with the fact that the coefficients on the same harmonics should be equal, the frequency-amplitude relationship can be obtained:

$$
\begin{aligned}
-\Omega^{2} A+\frac{3}{4} n_{1} A^{3}+n_{3} A & =\widehat{F}_{e} \cos \theta \\
-2 \varsigma \Omega A & =\widehat{F}_{e} \sin \theta
\end{aligned}
$$

Adding the square of left and right side of above two equations can obtain

$$
\left(-\Omega^{2} A+\frac{3}{4} n_{1} A^{3}+n_{3} A\right)^{2}+(-2 \varsigma \Omega A)^{2}=\widehat{F}_{e}^{2} .
$$

The solution of the system under forced excitation can be obtained by solving (16). The nondimensional force of the system after the vibration absorber can be expressed as

$$
f_{t}=2 \varsigma \widehat{u}^{\prime}+n_{1} \widehat{u}+n_{3} \widehat{u}^{3} .
$$

Applying HB method and assuming that the nondimensional solution form as $\widehat{f}_{t}=\widehat{F}_{t} \cos \left(\Omega \tau+\varphi_{T}\right)$, the magnitude of the force is given by

$$
\widehat{F}_{t}=A \sqrt{\left(n_{1}+\frac{3}{4} n_{3} A^{2}\right)^{2}+4 \varsigma^{2} \Omega^{2}}
$$

thus the force transmissibility is given by [10]

$$
\left|T_{F}\right|=\frac{\left|\widehat{F}_{t}\right|}{\left|\widehat{F}_{e}\right|}=\frac{\left|A \sqrt{\left(n_{1}+(3 / 4) n_{3} A^{2}\right)^{2}+4 \varsigma^{2} \Omega^{2}}\right|}{\left|\widehat{F}_{e}\right|} .
$$

Based on (19), the vertical vibration force transmissibility of the new vibration absorber is shown in Figure 5, in which the dash line is an unstable solution. It can be seen from the figure that with the increasing of the excitation amplitude, the resonance frequency and amplitude of the new vibration absorber transmissibility increase.

\section{Establishing Simulation Model}

As the studies of high-speed train vehicle dynamics systems go on, simply considering car body as a rigid body cannot reflect the elastic vibration of the car body. To solve this problem, the local flexible vibration of the car body needs to be taken into consideration. The flexible system considers the coupling vibration between the flexible body and the whole system in comparison to the original rigid system [11]. It makes the virtual prototype simulation more similar to the

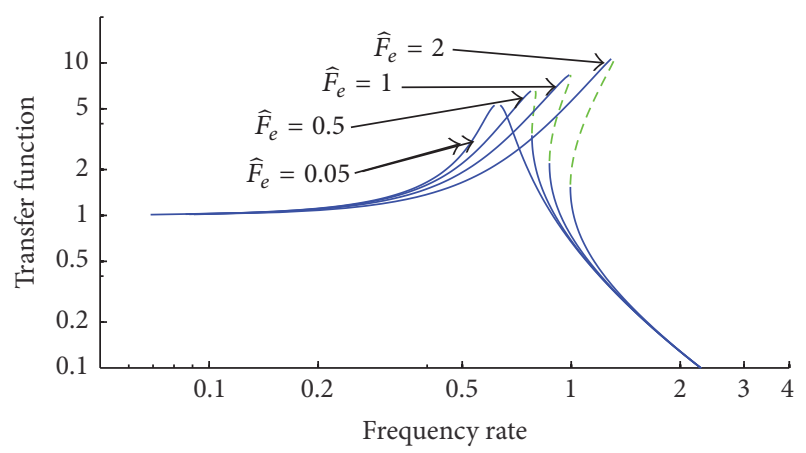

FIgURE 5: Vertical force transmissibility.

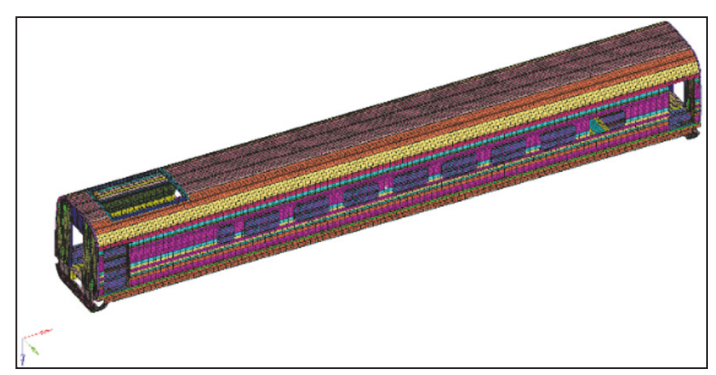

Figure 6: The finite element model of car body.

TABLE 1: The car body eigenfrequency results.

\begin{tabular}{lcc}
\hline Order & Mode shape & Frequency/Hz \\
\hline 1 & Diagonal distortion & 9.701 \\
2 & Vertical bending & 11.625 \\
3 & Torsion & 12.421 \\
4 & Lateral bending & 14.163 \\
5 & Breathing & 14.353 \\
\hline
\end{tabular}

real situation. In this paper, a rigid coupling dynamic model of the high-speed train considering the under-chassis device is established by SIMPACK multibody dynamics simulation software.

A finite element model of the vehicle body needs to be established in order to obtain the rigid-flexible coupling dynamics model, including the elastic body. The polycondensation theory is used to carry out the polycondensation of the vehicle body finite element model [12]. The model file contains the body structure and modal information through the SIMPACK software FEMBS interface into the dynamic model. The finite element model of the car body contains 630191 nodes and 775146 elements, which is shown in Figure 6. The frequency results of the first five modes of the car body are shown in Table 1.

A rigid-flexible coupling dynamics model for a complete vehicle system is established, which includes 1 car body, 1 under-chassis device, 2 bogies, 8 axle boxes, and 4 wheelsets. Three dimensions are considered in ever rigid body, and the total rigid degrees of freedom of the model are 48 . Compared with the elastic car body, the device, bogies, axles, 


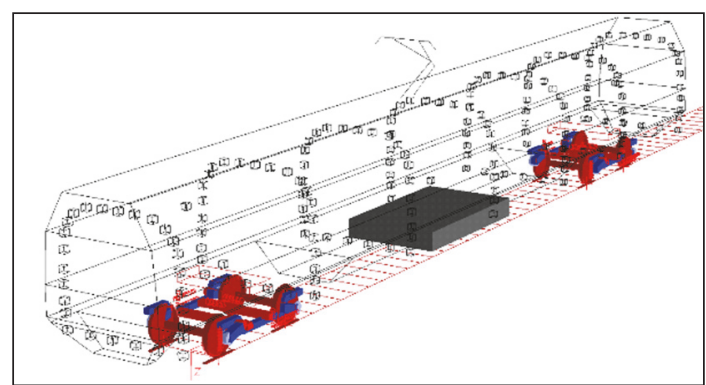

FIGURE 7: Rigid-flexible coupled dynamic system model.

TABle 2: Dynamic parameters of the car body.

\begin{tabular}{lcc}
\hline Item & Value & Unit \\
\hline Car body mass & 32 & $\mathrm{t}$ \\
Car body side-rolling vibration inertia & 120 & $\mathrm{t} \cdot \mathrm{m}^{2}$ \\
Car body pitching vibration inertia & 1740 & $\mathrm{t} \cdot \mathrm{m}^{2}$ \\
Car body yawing vibration inertia & 1670 & $\mathrm{t} \cdot \mathrm{m}^{2}$ \\
Frame mass & 3.3 & $\mathrm{t}$ \\
Frame side-rolling vibration inertia & 1.800 & $\mathrm{t} \cdot \mathrm{m}^{2}$ \\
Frame pitching vibration inertia & 1.300 & $\mathrm{t} \cdot \mathrm{m}^{2}$ \\
Frame yawing vibration inertia & 2.700 & $\mathrm{t} \cdot \mathrm{m}^{2}$ \\
Secondary vertical stiffness (each) & 210 & $\mathrm{kN} / \mathrm{m}$ \\
Secondary lateral stiffness (each) & 150 & $\mathrm{kN} / \mathrm{m}$ \\
Secondary lateral damping ratio (each) & 15 & $\mathrm{kNs} / \mathrm{m}$ \\
Primary vertical stiffness (each) & 1200 & $\mathrm{kN} / \mathrm{m}$ \\
Primary vertical damping ratio (each) & 13 & $\mathrm{kNs} / \mathrm{m}$ \\
Half of the length between bogie pivot centers & 8.75 & $\mathrm{~m}$ \\
Half of wheelbase & 1.25 & $\mathrm{~m}$ \\
Total length of the car body & 24.5 & $\mathrm{~m}$ \\
Mass of under-chassis device & 6400 & $\mathrm{~kg}$ \\
\hline
\end{tabular}

and wheelsets are still considered as rigidity because of the small elastic deformation. According to the contribution of car body elasticity to vibration energy, car body elasticity only considers the main low-order elastic mode [13], as shown in Table 1. The nonlinear geometrical characteristics of the wheel-rail contact, the nonlinear creep, the nonlinear characteristics of the hydraulic vibration absorber, and the incremental stiffness properties of the second lateral stop are also considered in the model. Figure 7 shows the rigidflexible coupling dynamics model of the high-speed train, and its dynamic parameters are presented in Table 2. The under-chassis device is suspended under the middle of the car body. The nonlinear force between under-chassis device and car body is modeled as an "input function" in SIMPACK.

The vertical and lateral stiffness of different combinations are selected in order to optimize the best match of vertical and lateral stiffness values of under-chassis device. The cylindrical rubber is used, which is characterized by equal lateral stiffness and longitudinal stiffness. In the process of calculation, we consider the hanging device vertical hanging frequency $f_{z}$ and lateral hanging frequency $f_{y}$ as variables, and then the vertical, lateral, and horizontal stiffness of the under-chassis devices $k_{z}, k_{y}$, and $k_{x}$ are, respectively,

$$
\begin{aligned}
& k_{z}=\left(2 \pi f_{z}\right)^{2} \times \frac{m}{n}, \\
& k_{y}=k_{x}=\left(2 \pi f_{y}\right)^{2} \times \frac{m}{n},
\end{aligned}
$$

where $m$ is mass of under-chassis device and $n$ is the number of hanging points. Each rubber has different damping ratios. If the damping ration of the rubber is too large, the aging creep can be accelerated because it is easy to heat. Usually damping ratio of natural rubber is $0.05 \sim 0.075$ [14], and this study selected damping ratio of 0.06 .

\section{Calculation and Analysis of the Optimum Value of Hanging Stiffness of the Under-Chassis Device}

The mass of under-chassis device is $m$, hanging stiffness is $k_{z}$, and the hanging frequency $f_{z}$ and static deflection $\delta_{\text {st }}$ can be obtained, respectively [15], as

$$
\begin{aligned}
& f_{z}=\frac{1}{2 \pi} \sqrt{\frac{k_{z}}{m}} \\
& \delta_{\mathrm{st}}=\frac{m g}{k_{z}}=\frac{g}{\left(2 \pi f_{z}\right)^{2}} .
\end{aligned}
$$

The stiffness and static deflection are determined by the hanging frequency. The static deflection of the device will be too large if the hanging frequency is too low, resulting in the device being beyond the boundary and causing other issues. Therefore, this study selected the frequencies range which are $4 \sim 16 \mathrm{~Hz}$.

The vehicle speed is $350 \mathrm{~km} / \mathrm{h}$ during the simulation process and the track irregularity adopts the low excitation high-speed spectrum [16]. The Sperling index of the vehicle at leading bogie, rear bogie, and middle part of the body is calculated. The results are shown in Figure 8(a) which shows the influence of the device hanging frequency on the lateral Sperling indexes (the average of the lateral Sperling index of the leading, rear bogies and the middle body of the car body). As can be seen from Figure 8(a), when the hanging frequencies are vertical $7 \mathrm{~Hz}$ and lateral $13 \mathrm{~Hz}$, the ride quality of the vehicle is the best; Figure $8(\mathrm{~b})$ is the influence of the device hanging frequency on the vertical Sperling indexes; when the device hanging frequencies are vertical $9 \mathrm{~Hz}$ and lateral $12 \mathrm{~Hz}$, the ride quality of the vehicle is the best; Figure 8(c) is the mean value of Sperling indexes on the vertical and lateral; when the device hanging frequencies are vertical $9 \mathrm{~Hz}$ and lateral $12 \mathrm{~Hz}$, the ride quality of the vehicle is the best. From Figures 8(a)-8(c), for Sperling index, the vertical hanging frequency of the device is more sensitive than the lateral; when the device hanging frequencies are vertical $9 \mathrm{~Hz}$ and lateral $12 \mathrm{~Hz}$, the vibration of the car body is the minimum, and the ride quality of the vehicle is the best. This is because the vertical bending frequency of the body of the body in this study is $11.6 \mathrm{~Hz}$, with a vertical hanging 


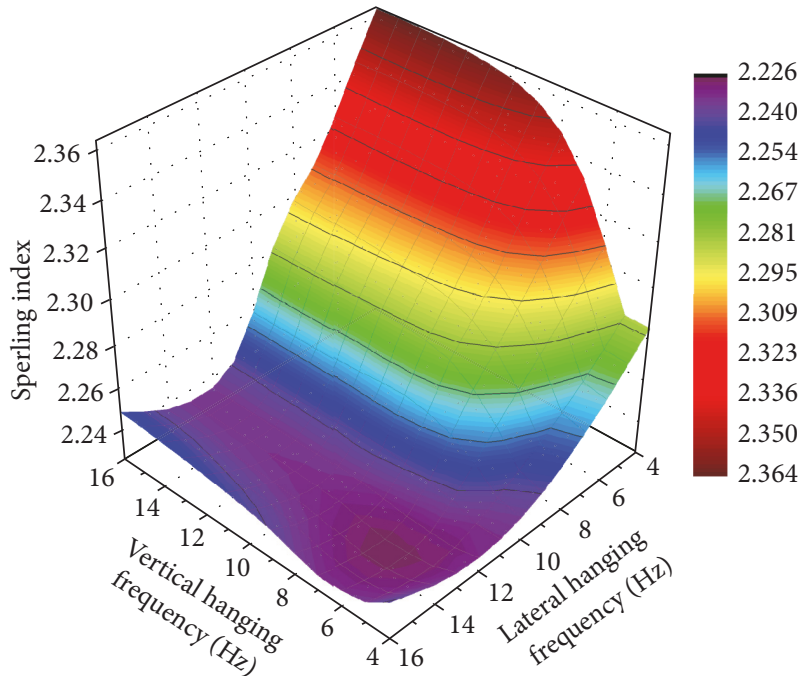

(a) Influence of device hanging frequency on lateral Sperling indexes

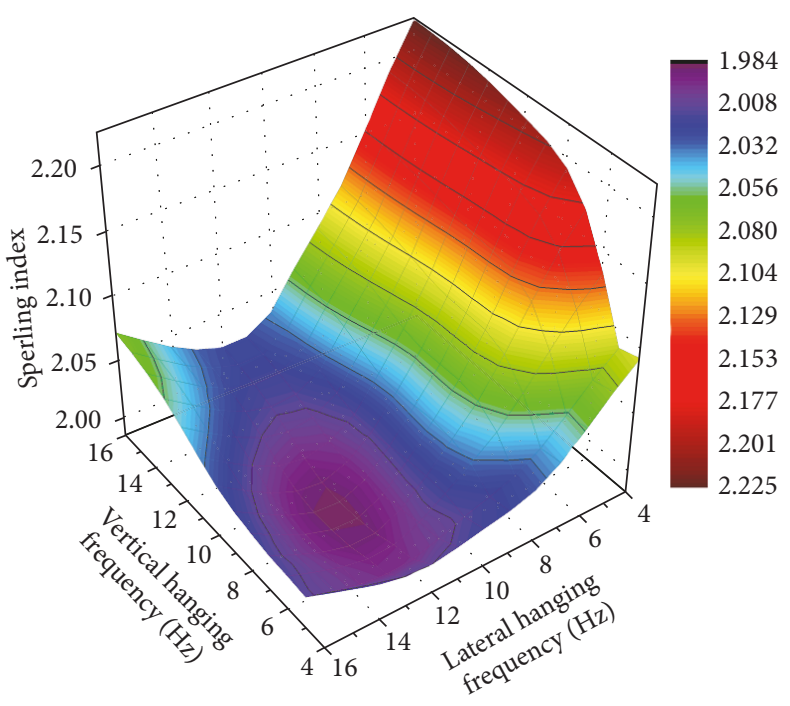

(b) Influence of device hanging frequency on vertical Sperling indexes

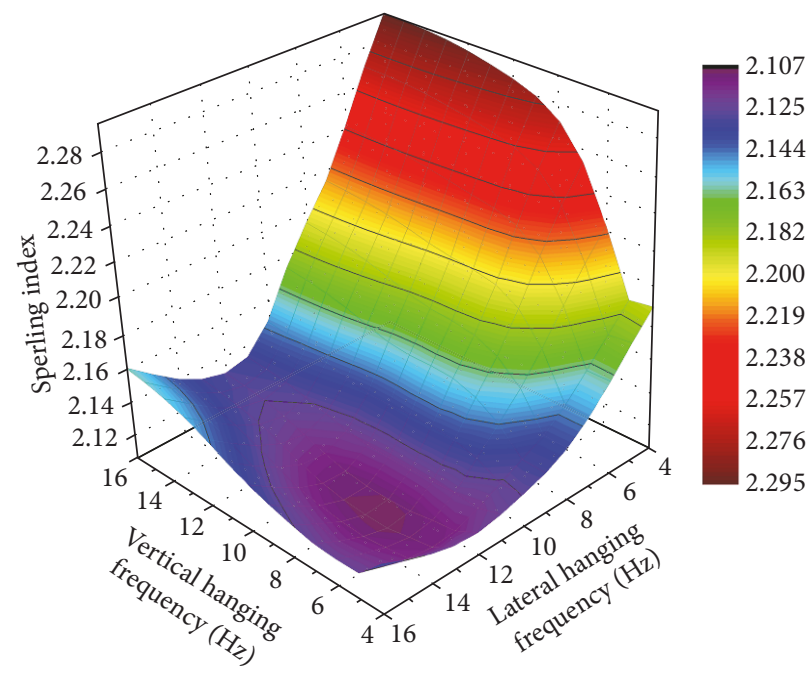

(c) Influence of device hanging frequency on the mean Sperling indexes of vertical and lateral

Figure 8

frequency of $9 \mathrm{~Hz}$ close to the optimal dynamic vibration absorber of the car body [17].

\section{Parameter Design of the Newly Vibration Absorber}

Through the analysis of the previous section, the optimal value of the device hanging frequencies is vertical $9 \mathrm{~Hz}$ and lateral $12 \mathrm{~Hz}$, and the mass of the device is $6400 \mathrm{~kg}$. If four hanging points are adopted, the target stiffness value of per hanging point can be given by

$$
\begin{aligned}
k_{z \text { Ideal }} & =(2 \pi \times 9)^{2} \times \frac{6400}{4}=5.12 \times 10^{6} \mathrm{~N} / \mathrm{m}, \\
k_{y \text { Ideal }} & =k_{x \text { Ideal }}=(2 \pi \times 12)^{2} \times \frac{6400}{4} \\
& =9.10 \times 10^{6} \mathrm{~N} / \mathrm{m} ;
\end{aligned}
$$

thus, the stiffness of the rubber spring for new vibration absorber can be given by

$$
\begin{aligned}
& k_{y}=k_{y \text { Ideal }}, \\
& k_{x}=k_{y} .
\end{aligned}
$$

The vertical stiffness of the rubber spring for the new vibration absorber can be obtained according to the verticallateral ratio of the rubber spring

$$
k_{z}=4.5 \times k_{y}=4.09 \times 10^{7} \mathrm{~N} / \mathrm{m} .
$$

Then, the negative stiffness of the disc spring for the new vibration absorber in the equilibrium position can be given by

$$
k_{d}(u=0)=k_{z \text { Ideal }}-k_{z}=-3.58 \times 10^{7} \mathrm{~N} / \mathrm{m} .
$$




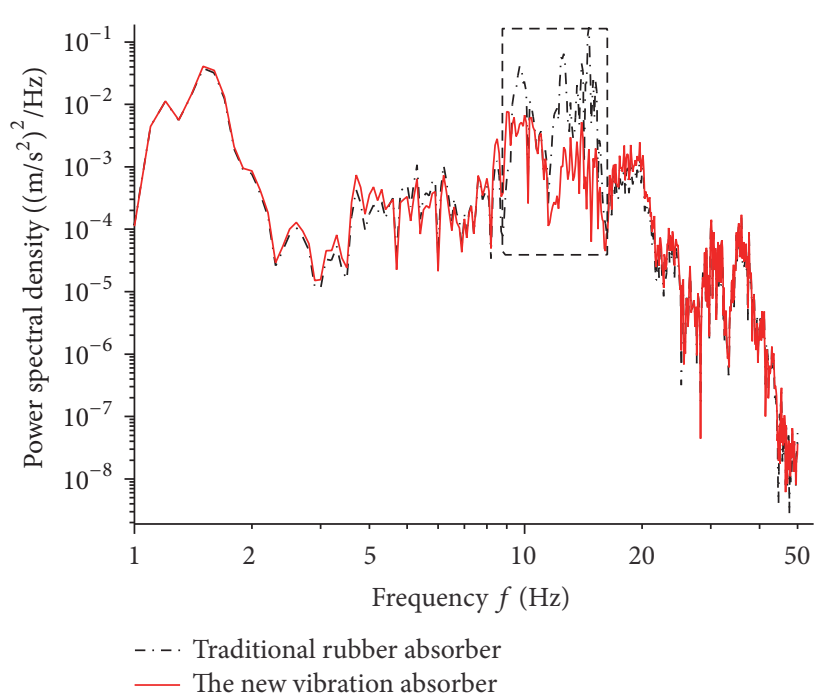

(a) Lateral acceleration power spectral density

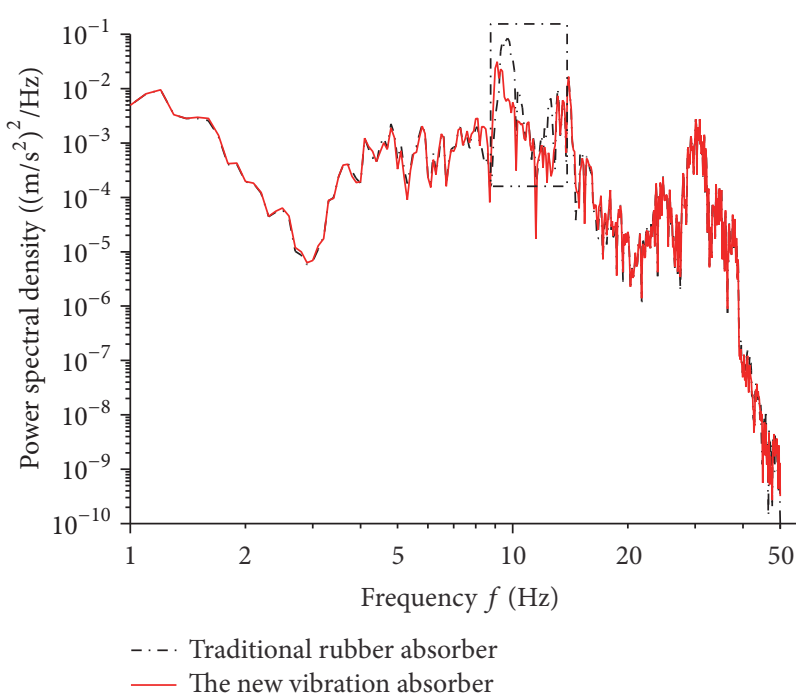

(b) Vertical acceleration power spectral density

Figure 9

TABLE 3: Parameters for disc spring.

\begin{tabular}{lccc}
\hline Parameters & Symbol & Unit & Value \\
\hline Elastic modulus & $E$ & $\mathrm{GPa}$ & 206 \\
Poisson ratio & $\mu$ & - & 0.03 \\
High thickness ration & $\alpha$ & - & 4 \\
Initial height & $h_{0}$ & $\mathrm{~mm}$ & 12.27 \\
Thickness & $t_{c}$ & $\mathrm{~mm}$ & 3.07 \\
Outside-inside diameter ratio & $C$ & - & 2 \\
Outside diameter & $D$ & $\mathrm{~mm}$ & 85.78 \\
Inside diameter & $d$ & $\mathrm{~mm}$ & 42.89 \\
\hline
\end{tabular}

Substituting (25) into (4), and combine with (9), the height parameter of the disc spring can be given. Assuming $\alpha=4$ and $C=2$, the parameters of the disc spring can be determined, as listed in Table 3 .

\section{Comparison of Simulation Result}

In order to study the vibration reduction effect of the new vibration absorber, the running vibration performance of the vehicle with the new vibration absorber and the vehicle with the traditional rubber absorbers is compared. The traditional rubber absorber is designed with the vertical stiffness value as the target value, and its lateral stiffness is calculated according to the vertical-lateral stiffness ratio

$$
\begin{aligned}
& k_{z}=k_{z \text { Ideal }}=(2 \pi \times 9)^{2} \times \frac{6400}{4}=5.12 \times 10^{6} \mathrm{~N} / \mathrm{m}, \\
& k_{y}=k_{x}=\frac{k_{z}}{4.5}=1.14 \times 10^{6} \mathrm{~N} / \mathrm{m} .
\end{aligned}
$$

Table 4 shows the Sperling indexes of the vehicle with the new vibration absorber and the vehicle with the traditional rubber absorber in the speed of $350 \mathrm{~km} / \mathrm{h}$. The results show that the Sperling indexes of the vertical and lateral of the vehicle with the new vibration absorber are significantly better than the vehicle with traditional rubber absorber.

Figures 9(a) and 9(b) show the results of vibration acceleration power spectral density (PSD) of the car body middle in comparison of the vehicle with the new vibration absorber and the vehicle with the traditional rubber absorber. As can be seen from the figure, the main energy of the lateral vibration acceleration is concentrated on the frequency range of $9 \sim 16 \mathrm{~Hz}$, and the vertical vibration acceleration is concentrated on the frequency range $9 \sim 12 \mathrm{~Hz}$. The acceleration PSD of the vehicle with the new vibration absorber is significantly better than the vehicle with traditional rubber absorbers in the concentrate frequency range. The peak value at $14 \mathrm{~Hz}$ for the lateral acceleration PSD and that at $9.7 \mathrm{~Hz}$ for the vertical acceleration PSD have been decreased, 95.8\% and 63.5\%, respectively, after adopting the new vibration absorber. The new vibration absorber can improve ride quality of the vehicle by effectively reducing the elastic vibration of the car body.

\section{Conclusion}

In this paper, a new vibration absorber is proposed based on the negative stiffness of the disc spring, which by the parallel method of rubber spring and disc spring can realize the separation of the vertical and lateral hanging stiffness. A rigid-flexible coupling dynamics model of high-speed train with elastic car body is established, and the optimum design of vertical and lateral hanging frequency is also taken into consideration. The optimal vertical and lateral suspension frequencies of the under-chassis device in this study are calculated, which are $9 \mathrm{~Hz}$ and $12 \mathrm{~Hz}$, respectively. The Sperling index, acceleration PSD of the vehicle with the new vibration absorber and the vehicle with traditional rubber absorber are compared and analyzed. The results show that, with the new vibration absorber, the vehicle's running stability and 
TABLE 4: Influence of two vibration absorbers on Sperling index of vehicle operation.

\begin{tabular}{lcccc}
\hline \multirow{2}{*}{ Position } & \multicolumn{2}{c}{ Lateral } & \multicolumn{2}{c}{ Vertical } \\
& Traditional rubber absorber & The new vibration absorber & Traditional rubber absorber & The new vibration absorber \\
\hline Running bogie & 2.3023 & 2.1733 & 2.1834 & 1.9671 \\
Middle & 2.4841 & 2.3765 & 2.1194 & 2.0076 \\
Rear bogie & 2.4055 & 2.1122 & 2.2869 & 1.9789 \\
Mean value & 2.3973 & 2.2207 & 2.1966 & 1.9845 \\
\hline
\end{tabular}

vibration of the car body are more effective than when using the traditional rubber absorber. The new vibration absorber can effectively reduce the elastic vibration of the car body and improve the ride quality of the vehicle.

\section{Conflicts of Interest}

The authors declare that there are no conflicts of interest regarding the publication of this paper.

\section{Acknowledgments}

The authors acknowledge the financial help provided by the Ministry of Science and Technology under the National Science and Technology Support Program Project (Grants nos. 2015BAG19B02 and 2015BAG13B01).

\section{References}

[1] P. Carlbom, Car body and passengers in rail vehicle dynamics [Ph.D. Thesis], Royal Institute of Technology (KTH), Stockholm, Sweden, 2000.

[2] E. Foo and R. M. Goodall, "Active suspension control of flexiblebodied railway vehicles using electro-hydraulic and electromagnetic actuators," Control Engineering Practice, vol. 8, no. 5, pp. 507-518, 2000.

[3] G. Diana, F. Cheli, S. Brunt, and A. Collina, "Dynamic Interaction between Rail Vehicles and Track for High Speed Train," Vehicle System Dynamics, vol. 24, no. 1, pp. 15-30, 1995.

[4] T. H. Young and C. Y. Li, "Vertical vibration analysis of vehicle/imperfect track systems," Vehicle System Dynamics, vol. 40, no. 5, pp. 329-349, 2003.

[5] W. Sun, D. Gong, J. Zhou, and Y. Zhao, "Influences of suspended equipment under car body on high-speed train ride quality," Procedia Engineering, vol. 16, pp. 812-817, 2011.

[6] D. Gong, J.-S. Zhou, and W.-J. Sun, “On the resonant vibration of a flexible railway car body and its suppression with a dynamic vibration absorber," Journal of Vibration and Control, vol. 19, no. 5, pp. 649-657, 2013.

[7] Z. Yinghui, Spring Manual, China Machine Press, Beijing, China, 1997.

[8] L. R. G. Treloar and D. J. Montgomery, The Physics of Rubber Elasticity, The Clarendon Press, 1958.

[9] I. Kovacic and M. J. Brennan, The Duffing Equation: Nonlinear Oscillators and Their Behaviour, 2011.

[10] L. Meng, J. Sun, and W. Wu, "Theoretical design and characteristics analysis of a quasi-zero stiffness isolator using a disk spring as negative stiffness element," Shock and Vibration, vol. 2015, Article ID 813763, 19 pages, 2015.
[11] D. Younesian, S. R. Marjani, and E. Esmailzadeh, "Importance of flexural mode shapes in dynamic analysis of high-speed trains traveling on bridges," Journal of Vibration and Control, vol. 20, no. 10, pp. 1565-1583, 2014.

[12] R. J. Guyan, "Reduction of stiffness and mass matrices," AIAA Journal, vol. 3, no. 2, pp. 1133-1145, 1965.

[13] P. F. Carlbom, "Combining MBS with FEM for Rail Vehicle Dynamics Analysis," Multibody System Dynamics, vol. 6, no. 3, pp. 291-300, 2001.

[14] W. Liang, Study of Shock Absorbing Rubber Material, Northwestern Polytechnical University, Shanxi, China, 2005.

[15] J. Trampe Broch, Mechanical Vibration and Shock Measurements, Bruel \& Kjaer, 1980.

[16] V. K. Garg and R. V. Dukkipati, Dynamics of Railway Vehicle Systems, Academic Press, New York, NY, USA, 1984.

[17] K. Seto, Dynamic Vibration Absorber and Its Applications, China Machine Press, 2013. 


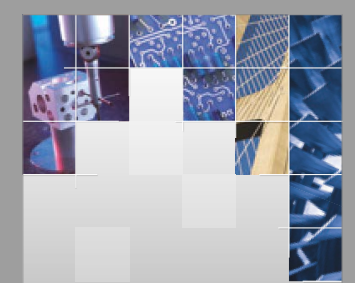

\section{Enfincering}
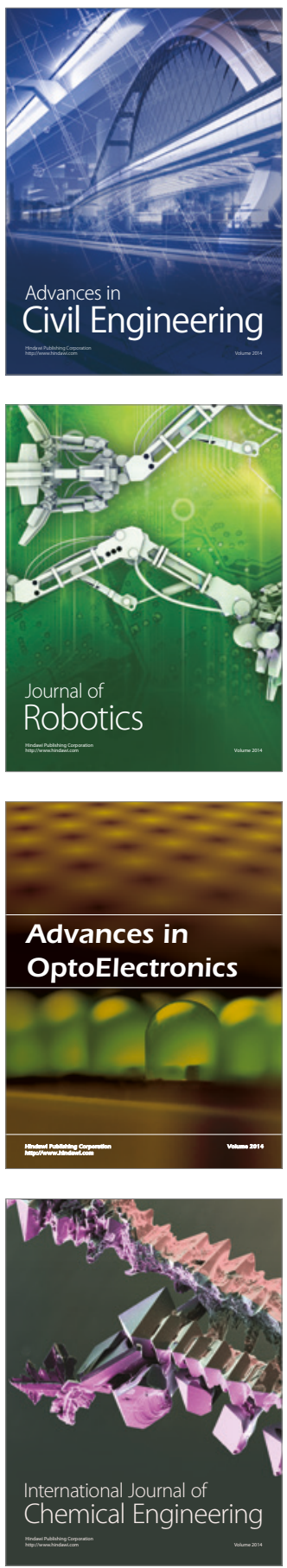

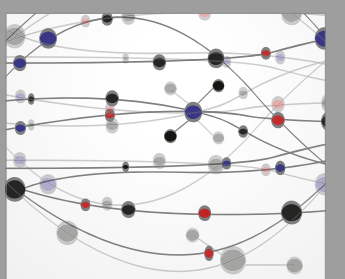

The Scientific World Journal

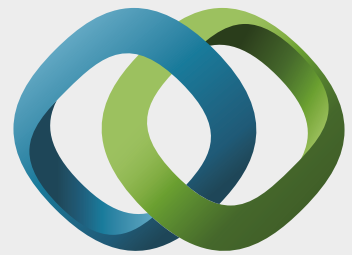

\section{Hindawi}

Submit your manuscripts at

https://www.hindawi.com
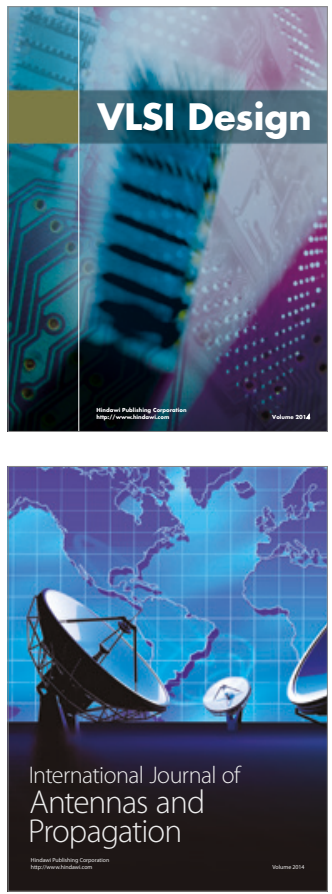

\section{Rotating}

Machinery
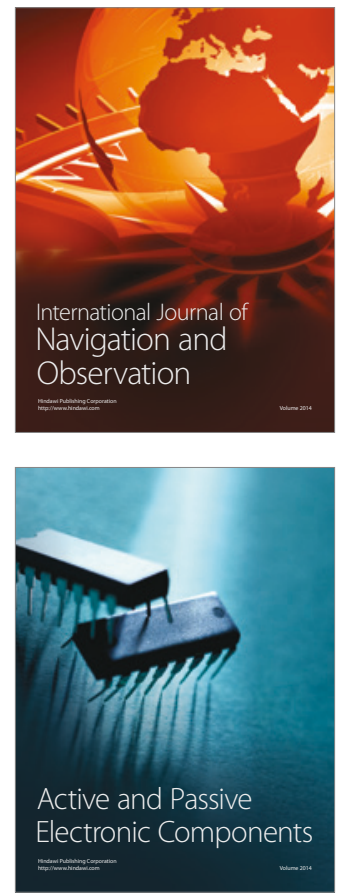
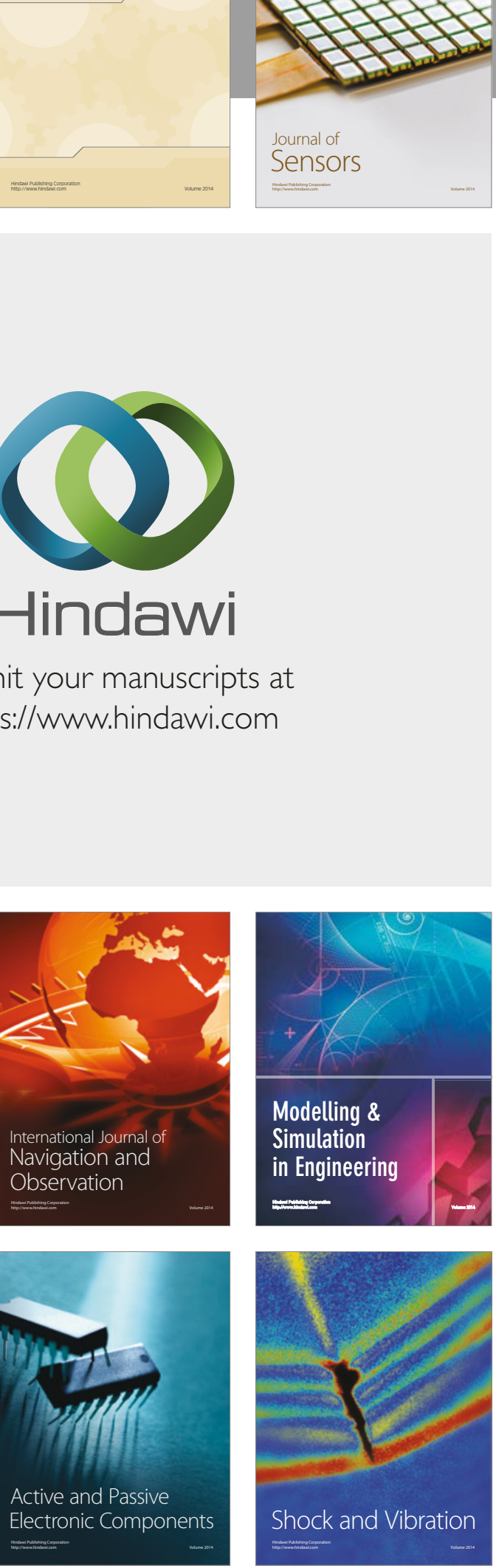
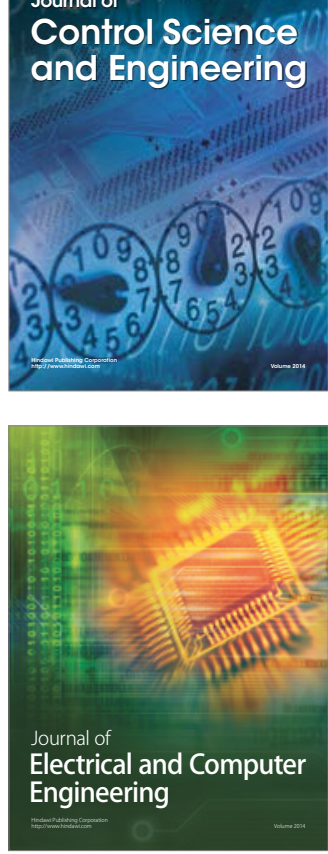

Distributed

Journal of

Control Science

and Engineering
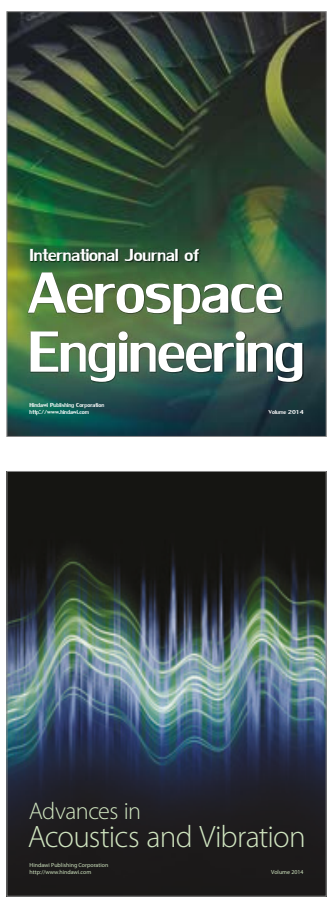

Sensor Networks 